\title{
Maximizing Seller's Profit for Electronic Commerce
}

\author{
Bella Belegradek ${ }^{1,3}$, Konstantinos Kalpakis ${ }^{1}$, and Yelena Yesha ${ }^{1,2,3}$ \\ 1 Computer Science and Electrical Engineering Department, University of Maryland \\ Baltimore County, 1000 Hilltop Circle, Baltimore, MD 21250. E-mail: \\ bbeleg1,kalpakis, yeyesha@cs.umbc.edu \\ 2 Center of Excellence in Space Data and Information Sciences, NASA Goddard \\ Flight Space Center, Greenbelt, MD 20771 \\ 3 Supported in part by the Center for Advanced Studies, IBM Toronto Laboratory
}

\begin{abstract}
Suppose there is a seller that has an unlimited number of units of a single product for sale. The seller at each moment of time posts a price for his/her product. Based of the posted price, at each moment of time, a buyer decides whether or not to buy a unit of that product from the seller. The only information about the buyer to the seller is the seller's sales history. Further, we assume that the maximal unit price the buyer is willing to pay does not change over time. The question then is how should the seller price his/her product to maximize profits?

To address this question, we use the notion of loss functions. Intuitively, a loss function is a measure, at each moment of time, of the lost opportunity to make a profit. In particular, we provide a polynomial-time algorithm that finds a pricing algorithm (strategy) for the seller that minimizes the average (total) losses over time. Further, we present preliminary results on pricing strategies that minimize the maximum possible loss at every moment of time. We also show that there is no strategy minimizing both the total loss and the maximum loss at the same time.
\end{abstract}

Keywords: Pricing strategies, market models, electronic commerce, computational learning theory, dynamic programming, search trees, discrete algorithms.

\section{Introduction}

We consider the following real-life problem: how should one price his or her products or services to maximize profits, when one does not know, ahead of time, the price one's customers are willing to pay? This problem becomes more important, given the recent explosion of interest in electronic commerce. In this paper, we provide optimal pricing strategies for the seller to maximize his/her average profits in a simplified market model that captures the essence of electronic commerce markets. 
Consider the following simple market model consisting of a seller and a buyer. The seller has an unlimited number of units of a single product for sale. At every moment of time ${ }^{1}$, the seller posts the current unit price for that product. At every moment of time, the buyer can buy a single unit of the product, if its unit price is no more than the price the buyer is willing to pay per unit, i.e. the buyer's valuation. When this happens, we say that a deal occurred at that time. Further, we assume that the seller never posts a price below the product's unit cost and that the only information available to the seller is the history of the seller's previous sales - the price set at any previous moment of time and whether a deal occurred at that time. In this paper, we also assume that the maximum unit price the buyer is willing to pay does not change over time. This market model is an approximation of the manner in which commerce is expected to be conducted over the Internet.

Generally, the buyer's valuation varies over time, reflecting numerous aspects of real life (i.e. the scarcity of the product in the market, subjective preferences of buyers, etc). In this paper, we consider a special case of buyer's valuation function, namely when the buyer's valuation is a constant in a given range, and the seller knows only that range of values. Hereafter, in order to simplify the discussion, and since the seller never sells below cost, we will refer to the difference between the maximum price the buyer is willing to pay for a unit of the product and the cost of the unit to the seller as the buyer's valuation. Then, the buyer's valuation is an integer constant, known only to the buyer at time 0 , in a range $[0, \ldots, N]$, where the integer $N$ is known to the seller. Note that $N$ can be an overestimate of the buyer's valuation by the seller.

The main question then is "what is the best pricing strategy for the seller?". In other words, how should the seller choose the unit price at a given moment of time in order to maximize profits?

Similar models are well known in economics of monopolistic pricing (see [AD89], [Ben89], [HR81], [Mey75], [Sob91]), All of them consider the market situation with a single seller and single or multiple buyers. However, they focus on comparing of various mechanisms of sales such as auctions, different prices set for different buyer groups or single set price ([HR81]); or on the existence of equilibria ${ }^{2}$ for the models they study.

L.M. Ausabel and R.J. Deneckere [AD89] and, later, J. Sobel [Sob91] for a more general model proved that there are equilibria for all levels of seller profits, if the buyers have an access to the sales history and are capable of strategic behavior.

R. Benabou in [Ben89] studied equilibria and optimal pricing strategies for the seller in inflationary monopolistic economics, while assuming the strategic behavior of the buyers and their knowledge of the sales history.

\footnotetext{
${ }^{1}$ We assume that time is measured in indivisible discrete units numbered by nonnegative integers. Further, we assume that both prices and buyer's valuations are non-negative integers.

${ }^{2}$ An equilibrium is a stable state in an economic system, such that a deviation from this state leads to a loss for the deviating side.
} 
Our approach is to use methods of computer science to create and analyze computationally effective algorithms for dynamic price changes that provide optimal profits for the seller. There is a number of recent works in theoretical computer science (see, for example, [AA95], [EYFKT92]) which use the competitive analysis of algorithms to solve optimization problems arising from economic problems. However the competitive analysis of algorithrns produces too coarse results to be used in practice (within a factor from the optimal off-line solution).

Learning theory seems to be more useful for these problems, especially when there are no time bounds, i.e. in optimization problems with infinite horizon. We use the notion of loss functions to choose an optimal pricing strategy for the seller. Intuitively, a loss function is a measure, at each moment of time, of the lost opportunity to make a profit. It is clear then that in order to maximize profits, one should minimize some loss function over time. In this paper, we provide a polynomial-time algorithm that finds a pricing algorithm (strategy) for the seller that minimizes the average (total) losses over time. Further, we present preliminary results on pricing strategies that minimize the maximum possible loss at every moment of time. We also show that there is no strategy minimizing both the total loss and the maximum loss at the same time.

The rest of this paper is organized as follows. In Sect. 2 we provide some preliminaries. Then, in Sect. 3, we provide an algorithm for computing a pricing strategy for minimizing the total loss. In Sect. 4, we provide lower and upper bounds on the maximum loss. Section 5 concludes this paper.

\section{Preliminaries}

Our goal is to choose an optimal seller's pricing algorithm for a given loss function and an interval $[0, \ldots, N]$ of possible buyer's valuations (or maximum buyer's prices). Recall that, in this paper, the maximum buyer's price reflects not the actual price, but rather an additional amount, the buyer is willing to pay over a fixed minimum seller's price.

The notion of "maximizing profit" is a little tricky in this case, as it often happens in many problems associated with on-line algorithms in general and on-line learning theory in particular. Clearly, there is no dominating algorithm that can provide the maximum achievable profit for any possible buyer's price.

The maximum profit in the worst case is 0 , and it does not depend on the pricing strategy. The worst case profit is always achieved for the lowest possible price and the best performing algorithm is the most conservative one, the one which starts with the lowest possible price and keeps increasing it by 1 for so long as a deal occurs. After the first time a deal does not occur, the seller returns to the previous price which is the true buyer's valuation. It is easy to see that this algorithm does not give us maximum profit when the buyer's valuation is close to its upper bound.

We use loss functions (frequently used in on-line learning theory), which provide another way to estimate the "goodness" of an algorithm, that unifies both the average-case and a sensible worst-case performance of that algorithm. 
The typical setting for an on-line learning problem includes the sequence of a learner's trials and the teacher's (or just the outer world's) responses, which are often the only input to a learning algorithm, to learn an unknown concept or distribution [Ben89,Vap96]. The closeness of the learner's perception of the target concept to the concept itself is measured by a loss function. Then, the best learning algorithm is the one that minimizes the value of that loss function over the space of all possible inputs.

Two types of loss functions are commonly used: (a) those in which the total loss of a sequence of trials is equal to the number of negative responses with all the responses are being binary[BDKM95,CBFHW93], and (b) those that are based on the distance, in some metric space, between the outer world sample and the estimate of this sample, predicted by the learning algorithm. In the latter case, the learner usually gets the sample itself as the teacher's response [KW93,Vap96]. We use hybrid loss functions.

Let $\mathcal{A}$ be a pricing algorithm, used by the seller. Let $X$ be the true maximum buyer's price, and let $P_{t}$ be the unit price at time $t$ set by a $\mathcal{A}$. The loss of $\mathcal{A}$ at time $t$ is the difference between the maximum profit the seller could get at time $t$ (which is equal to $X$ ) and the profit actually obtained at time $t$ :

$$
\text { loss }_{\mathcal{A}}(X, t)=\left\{\begin{array}{l}
X, \text { if } P_{t}>X \\
X-P_{t}, \text { otherwise }
\end{array}\right.
$$

The loss of $\mathcal{A}$ on input $X$ is

$$
\operatorname{loss}_{\mathcal{A}}(X)=\sum_{t=0}^{\infty} \operatorname{loss}_{\mathcal{A}}(X, t) .
$$

We consider the following two loss functions:

(a) The total loss of the pricing algorithm $\mathcal{A}$ : total_loss $_{\mathcal{A}}=\sum_{X=0}^{N} \operatorname{loss}_{\mathcal{A}}(X)$.

(b) The maximum loss of the pricing algorithm $\mathcal{A}$ : $\max _{-} \operatorname{loss}_{\mathcal{A}}=\max _{0 \leq X \leq N} \operatorname{loss}_{\mathcal{A}}(X)$.

Note that total_loss $s_{\mathcal{A}}$ is the average-case loss multiplied by $N$ when $X$ is uniformly distributed on $[0, N]$, and that $\max _{-} l o s s_{\mathcal{A}}$ is the worst-case loss.

Obviously, if the seller knows $X$ at time $\tau$, then, by setting $P_{t}=X$ for each $t \geq \tau$, the seller will not incur any losses after time $\tau$. Therefore, if a pricing algorithm $\mathcal{A}$ is able to recognize any possible maximum buyer's price $X$ in a finite amount of time $t^{\prime}$, then, by setting $P_{t}=X$ for all $t \geq t^{\prime}$, the $\operatorname{loss}_{\mathcal{A}}(X)$ is finite for every $X \in[0, \ldots, N]$. Thus in order for a pricing algorithm to be optimal with respect to any of the two loss functions defined above, such an algorithm must be able to find the maximum buyer's price in a finite amount of time and must provide finite losses on all possible inputs in a given range.

Given a pricing algorithm $\mathcal{A}$, let $\mathcal{P}_{t}(X)$ be a set of possible maximum buyer's prices as it is viewed by $\mathcal{A}$ at time $t$, when the true maximum buyer's price is $X$. Clearly, $\mathcal{P}_{0}(X)=[0, \ldots, N]$ for any $X$, while $\mathcal{P}_{t}(X)$ depends on $X$ and 
the prices set by $\mathcal{A}$ until time $t$. Further, $X \in \mathcal{P}_{t}(X)$ for all $t$. We call a pricing algorithm $\mathcal{A}$ admissible, if for all $X \in[0, \ldots, N]$ and every time $t$ either $\mathcal{P}_{t}(X)=\{X\}$ or $\mathcal{P}_{t+1}(X) \subset \mathcal{P}_{t}(X)$. Intuitively, an admissible pricing algorithm does not ask unnecessary questions, thus it makes progress towards finding the maximum buyer's price at every time step. Algorithm $\mathcal{A}$ knows (learns or finds) the maximum buyer's price $X$ at time $\tau$, if $\mathcal{P}_{r}(X)=\mathcal{P}_{r+1}(X)=\cdots=\{X\}$.

Lemma 1. Let $\mathcal{A}$ be an admissible pricing algorithm for $[0, \ldots, N]$. Then, $\mathcal{A}$ can be represented as a binary search tree with $N+1$ leaves and $N$ internal nodes, each of degree 2. Furthermore, there exist admissible optimal pricing algorithms for both the total loss and the maximum loss functions.

Proof. Observe that $\mathcal{A}$, by setting the price $P_{t}$ to be some number $Y$, effectively asks the question "Is $Y \leq X$ ?". The occurrence of a deal at time $t$ is equivalent to an affirmative answer to this question. Thus, if $\mathcal{P}_{t}(X) \neq\{X\}$ then

$$
\mathcal{P}_{t+1}(X)=\left\{\begin{array}{l}
\left\{y: y \in \mathcal{P}_{t}(X) \text { and } y \geq P_{t}\right\}, \text { if } P_{t} \leq X, \\
\left\{y: y \in \mathcal{P}_{t}(X) \text { and } y<P_{t}\right\}, \text { if } P_{t}>X .
\end{array}\right.
$$

Since the occurrence or non-occurrence of a deal is the only new information made available to $\mathcal{A}$ at every moment of time, $\mathcal{A}$ is, in effect, a binary search on an interval. Every question corresponds to an internal node in a search tree $T$ of the algorithm and every leaf is a possible value of $X$. So every internal node in a tree representing $\mathcal{A}$ has degree 2 .

Consider now a pricing algorithm $\mathcal{A}^{\prime}$. If for some $X$ the set of possible values of $X$, as it is viewed by $\mathcal{A}^{\prime}$, is the same for several consecutive moments of time $t, t+1, \ldots, t+k$ and reduces at time $t+k+1$, then, the algorithm $\mathcal{A}^{\prime \prime}$, that "skips" all the (redundant) steps of $\mathcal{A}^{\prime}$ from time $t$ until time $t+k$ but is otherwise identical to $\mathcal{A}^{\prime}$, does not increase the losses for any $X$. Thus, $\mathcal{A}^{\prime \prime}$ is not worse than $\mathcal{A}^{\prime}$ with respect to any of our loss functions. Repeating this procedure for a finite number of times, we can obtain an admissible algorithm that provides at most the same values for the both loss functions as $\mathcal{A}^{\prime}$ does. Therefore, there always exists an optimal admissible algorithm for any of our loss functions.

By Lemma 1, we only need to consider admissible pricing algorithms when searching for optimal pricing algorithms. Furthermore, since to any admissible pricing algorithm there corresponds a binary search tree with $N+1$ leaves and $N$ internal nodes each of degree 2 , one could find an optimal pricing algorithm by an exhaustive search over all such binary search trees. However, since there are $\frac{1}{1+N}\left(\begin{array}{c}2 N \\ N\end{array}\right)$ such trees, such an approach is computationally infeasible for large values of $N$. Thus, we need an efficient way for search over the space of binary search trees in order to find optimal admissible pricing algorithms.

Let $T$ be the binary search tree representation of an admissible pricing algorithm $\mathcal{A}$ for $[0, \ldots, N]$. We cast the definition of loss functions in terms of the tree $T$. Consider a (unique) search path in $T$ from its root to the leaf labeled $X$. 
We denote this path by $y_{0}, y_{1}, \ldots, y_{k}$, where $y_{0}=\operatorname{root}(T)$ and $y_{k}$ is the leaf $X$. The loss of the search for $X$ on the edge $\left(y_{i}, y_{i+1}\right)$ or, alternatively, the loss of the search for $X$ at the $i$-th step in $T$ is

$$
\operatorname{loss}_{T}\left(X,\left(y_{i}, y_{i+1}\right)\right)=\left\{\begin{array}{l}
X, \text { if } X<y_{i} \\
X-y_{i}, \text { otherwise }
\end{array}\right.
$$

and the loss of the search for $X$ in $T$ is

$$
\operatorname{loss}_{T}(X)=\sum_{i=0}^{k-1} \operatorname{loss}_{T}\left(X,\left(y_{i}, y_{i+1}\right)\right) .
$$

The total loss total_loss $T(N)$ and maximum loss $\max _{\text {_loss }}(N)$ are defined as the sum and the maximum, respectively, of the individual losses for all $X \in$ $[0, \ldots, N]$. The goal is then, given the interval $[0, \ldots, N]$, construct a binary search tree $T_{\text {total }}\left(T_{\max }\right)$ that minimizes the total (maximum) loss among all possible binary search trees.

\section{Minimizing the Total Loss}

First, we compute the value of the total loss of the most natural search tree the (perfectly) balanced binary search tree. For simplicity, we compute the total loss for $N=2^{k}-1$, where $k$ is a non-negative integer. Although, as we prove later in this section, the balanced binary search tree does not minimize the total loss, the obtained value is useful as an upper bound on the total loss.

Lemma 2. The total loss of the (perfectly) balanced binary search tree for $[0, \ldots$, $\left.2^{k}-1\right]$ is equal to

$$
k\left(4^{k-1}-2^{k-1}\right)
$$

Proof. omitted. See full paper for a detailed proof.

Second, using dynamic programming [CLR.93], we provide a polynomialtime algorithm that finds an admissible pricing algorithm that minimizes the total loss.

Theorem 3. Given a positive integer $N$, we can find an admissible algorithm that minimizes the total loss on $[0, \ldots, N]$, in $O\left(N^{3}\right)$ time while using $O\left(N^{2}\right)$ space.

Proof. Let $T$ be a binary search tree for an interval $[i, \ldots, j]$ which provides the minimum total loss for this interval. Let $k$ be the root of $T$. Since $T$ is the representation of an algorithm that reduces the range of possible values of $X$ on every step, we conclude that $i+1 \leq k \leq j$.

Observe that for any $X<k, \operatorname{loss}_{T}(X)=\operatorname{loss}_{T_{L}}(X)+X$ and that for any $Y \geq k, \operatorname{loss}_{T}(Y)=\operatorname{loss}_{T_{R}}(Y)+Y-k$, where $T_{L}$ and $T_{R}$ are the left and right 
subtrees of the root of $T$ respectively. Therefore,

$$
\begin{aligned}
& \text { total_loss }_{T}(i, j)=\sum_{X=i}^{k-1}\left(\operatorname{loss}_{L}(X)+X\right)+\sum_{Y=k}^{j}\left(\operatorname{loss}_{R}(Y)+Y-k\right)= \\
& =\text { total_loss } T_{L}(i, k-1)+\text { total_loss } T_{R}(k, j)+\frac{(j-i+1)(j+i)}{2}-(j-k+1) k .
\end{aligned}
$$

Next, we show that the problem has the optimal sub-structure property [CLR93], i.e. the restriction of the optimal solution to any subproblem is the optimal solution of this subproblem. Suppose that $T_{L}$ does not provide the minimum total loss for the interval $[i, \ldots, k-1]$. Let $T_{L}^{*}$ be the optimal tree for this interval. Then, replacing $T_{L}$ with $T_{L}^{*}$ as the left subtree of the root of $T$, we get a tree for the interval $[i, \ldots, j]$ whose total loss is less than that of $T$. A contradiction. Similarly, $T_{R}$ has to minimize the total loss on the interval $[k, \ldots, j]$.

Let $T L(i, j)$ denote the minimum total loss on $[i, \ldots, j]$. Then, from (7) we get the following recurrence

$$
T L(i, j)=\min _{i+1 \leq k \leq j}\{T L(i, k-1)+T L(k, j)+(j-i+1)(j+i) / 2-(j-k+1) k\},
$$

where $T L(i, i)=0$. Using algorithm Total_Loss whose pseudo-code is given in figure 1 , we can compute $T L(0, N)$ in $O\left(N^{3}\right)$ time and $O\left(N^{2}\right)$ space. This algorithm uses tables $T L[0 . . N, 0 . . N]$ and $\operatorname{root}[0 . . N, 0 . . N]$ for storing $T L(i, j)$ and $\operatorname{root}(i, j)$ - the root of an optimal binary search tree for $[i, \ldots, j]$, respectively. The pseudo-code Optimal_Tree in figure 1 constructs an optimal tree $T_{\text {total }}$ for the interval $[i, j]$ in the additional time $O(j-i)$.

Corollary 4. The (perfectly) balanced binary search tree does not minimize the total loss.

Proof. We provide a counterexample. The minimum total loss for $N=7$, computed by Theorem 3 , is 33 , while the total loss of the balanced binary search on $[0, \ldots, 7]$ is 36 .

Third, we compute lower and upper bounds for the minimum total loss over the interval $[0, \ldots, N]$.

Lemma 5. Let $t l(N)$ denote the minimum total loss for $[0, \ldots, N]$. Then,

$$
\left\lfloor\frac{N}{2}\right\rfloor\left\lceil\frac{N}{2}\right\rceil \leq t l(N) \leq(\lfloor\log N\rfloor+1) 2^{\lfloor\log N\rfloor}\left(2^{\lfloor\log N\rfloor}-1\right) .
$$

Proof. From (8), since $t l(N)=T L(0, N)$, we have that

$$
t l(N) \geq \min _{1 \leq k \leq N}((N+1) N / 2-(N+1-k) k)=\left\lfloor\frac{N}{2}\right\rfloor\left\lceil\frac{N}{2}\right\rceil .
$$

Let $k=\lfloor\log N\rfloor+1$. Then, $2^{k-1} \leq N \leq 2^{k}-1$. Note that, since $t l(x)$ is a non-decreasing function of $x, t l(N) \leq t l\left(2^{\bar{k}}-1\right)$. On the other hand, if $T$ is a 
Total_Loss $(N)$

\section{BEGIN}

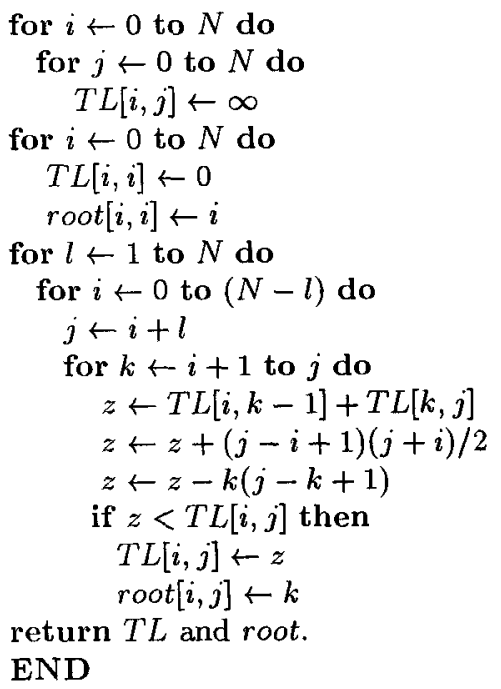

Optimal_Tree(int $i, j$, TreeNode $*$ node)

\section{BEGIN}

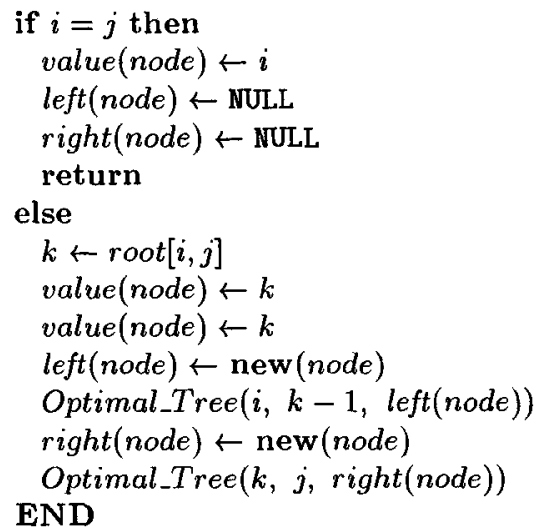

Fig. 1. Algorithms for finding and explicitly constructing binary search tree minimizing total loss.

balanced search tree on $\left[0, \ldots, 2^{k}-1\right]$, then $t l\left(2^{k}-1\right) \leq$ total loss $T$. Then, from (6) we have that

$$
\left.t l(N) \leq(\lfloor\log N\rfloor+1) 2^{\lfloor\log N\rfloor} 2^{\lfloor\log N\rfloor}-1\right) .
$$

Fourth, we generalize Theorem 3 to find an admissible pricing algorithm that minimizes the expected loss, when the maximum buyer's price $X$ has an arbitrary discrete probability distribution on $[0, \ldots, N]$.

Let the buyer's valuation $X$ be a discrete random variable with given probability mass function (p.m.f.) $-p_{X}(x)$.

The expected loss for a binary search tree $T$ on the interval $[0, \ldots, N]$ under the given distribution is

$$
\overline{\operatorname{loss}}(T)=\sum_{x=0}^{N} \operatorname{loss_{T}}(x) p_{X}(x)
$$

Theorem 6. Given a probability mass function of buyer's prices on $[0, \ldots, N]$, we can find an admissible algorithm that minimizes the expected loss on this interval, together with the expected loss achieved by that algorithm, in $O\left(N^{3}\right)$ time and using $O\left(N^{2}\right)$ space.

Proof. Similar to the proof of Theorem 3. See full paper for details. 


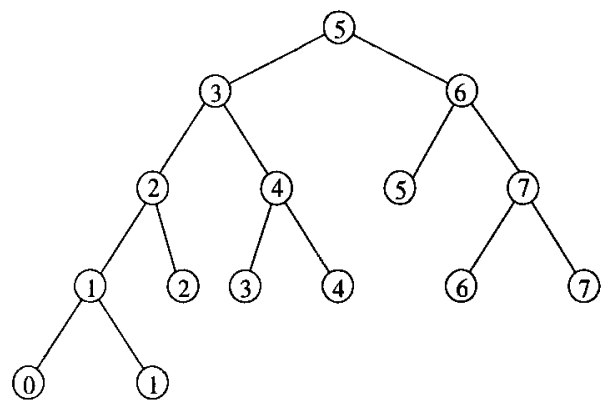

Fig. 2. Tree $T_{\text {lotal }}(7)$ that minimizes the total loss on $[0, \ldots, 7]$

In appendix A, we provide sample trees that minimize the expected loss for various probability distributions.

\section{Bounding the Maximum Loss}

Unlike the problem of computing the minimum total loss, the problem of computing the minimum maximum loss does not have the optimal sub-structure property. So, dynamic programming can not be used for this problem.

First, we compute lower and upper bounds for the minimum maximum loss as a function of $N$.

Lemma 7. Let $m l(N)$ denote the minimum maximum loss on $[0, \ldots, N]$. Then,

$$
N-1 \leq m l(N) \leq\lfloor\log N\rfloor\left(2^{\lfloor\log N\rfloor+1}-2\right) .
$$

Proof. Recall that each admissible algorithm is able to recognize every number in $[0, \ldots, N]$. Particularly, such an algorithm has to distinguish between $N-1$ and $N$. The only question that can achieve this is: "Is $N \leq X$ ?". The answer to this question is "Yes", if $X=N$, and is "No", if $X=N-1$. Thus, for any binary search tree $T, \operatorname{loss}_{T}(N-1) \geq N-1$.

Let $k=\lfloor\log (N)\rfloor+1$. Consider the (perfectly) balanced binary search tree $T$ on $\left[0, \ldots, 2^{k}-1\right]$. Let $k=\lfloor\log (N)\rfloor+1$. We compute an upper bound for the maximum loss on $T$ over the interval $\left[0, \ldots, 2^{k}-1\right]$ as follows:

$$
{\max \_l o s s_{T}}=\max _{0 \leq X \leq 2^{k}-1} \operatorname{loss}_{T}(X)=\max _{0 \leq X \leq 2^{k}-1} \sum_{i=0}^{k-1} \operatorname{loss}_{i}(X) \leq k\left(2^{k}-1\right),
$$

where $\operatorname{loss}_{i}(X)$ denotes the loss of the search for $X$ on the $i$-th step in $T$. To improve the upper bound we observe that, for every $X$, the maximum loss on 
the $i$-th step does not exceed $N-1=2^{k}-2$. Also for every $X$, there is a step at which no loss occurs. Then, since $\operatorname{loss}_{\mathcal{A}}(0)=0$ for any pricing algorithm $\mathcal{A}$, we have that $\max _{-} \operatorname{loss}_{T} \leq(k-1)\left(2^{k}-2\right)$.

Note that the maximum loss on a given interval, as the worst case measure, is the same for any probability distribution of the buyer's valuation on that interval.

Furthermore, the (perfectly) balanced binary search tree does not minimize the maximum loss. For example, the tree $T_{\text {total }}(15)$, that minimizes the total loss on the interval $[0, \ldots, 15]$, has maximum loss equal to 25 . However, the (perfectly) balanced binary search tree $T$ has maximum loss equal to 28 .

Theorem 8. There is no strategy that minimizes both the total loss and the maximum loss functions at the same time.

Proof. We give a proof by counterexample.

By performing an exhaustive search among all binary search trees, as stipulated in Lemma 1 , on $[0, \ldots, N]$, we found that for each $N, 1 \leq N \leq 8$, a tree that minimizes the total loss among all the trees that minimize the maximum loss. For $N=8$ the minimum maximum loss is equal to 8 , see figure 3 ; the minimum total loss among the trees with $\max _{-}$loss $=8$ is 47 . On the other hand, the minimum total loss on $[0, \ldots, 8]$, computed by the algorithm in figure 1 is 46 .

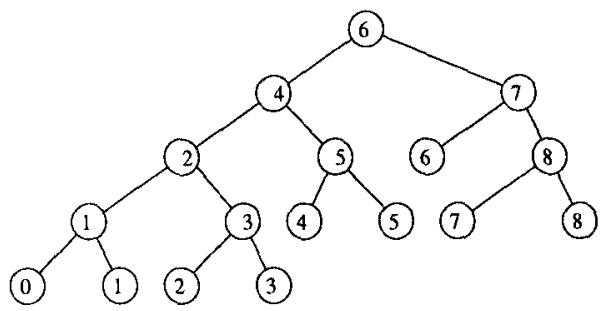

Fig. 3. Tree $T_{\max }(8)$ that minimizes the maximum loss on $[0, \ldots, 8]$ and has the minimum total loss among all those trees with the same maximum loss.

Therefore, minimizing the total loss does not necessarily minimize the maximum loss and vice versa.

\section{Conclusions}

We introduce a simple market model for buyer/seller sales transactions that captures the essence of electronic commerce over the Internet, and consider the problem of pricing algorithms for the seller that maximize his/her profits. Using the idea of loss functions, we provide a polynomial-time algorithm that computes a pricing strategy for the seller that minimizes the cumulative (total) loss (lost 
profit because no sale occurred), when the buyer's valuation is an unknown constant in a given range. We also compute lower and upper bounds on the total loss. Further, we present preliminary results for the problem of minimizing the maximum loss incurred at any time.

Some of the future work includes extending our simple market model and pricing strategies to the case of multiple buyers with valuations that vary over time, as well as resolving the problem of minimizing the maximum loss in our current model.

Finally, we would like to discuss another application for our work. Economic methods have been recently used as an extremely effective tool for resource allocation in distributed computer systems. The main advantages of market based models in computing are rooted in their inherently distributed and selfstabilizing nature. A vivid example of such an approach is Load Balancing Economy, described by Ferguson et. al. [FNSY96]. This economy consists of multiple processors, connected by point-to-point links with given service rates and propagation delays. The resources are CPU time and communication bandwidth. Jobs enter the distributed system and request services based on preferences price preference, time preference, or combined price-and-time preference. The processors advertise resource prices and each job computes the best node to be serviced at, based on its preferences, wealth and resource prices. Pricing mechanisms proposed for this model are several kinds of auctions.

Load Balancing Economy fit our model especially well, since the jobs, generated by the same source, tend to have equal budgets (valuaions), and since a job is usually not capable of strategic behavior. Therefore, we suggest to use dynamic pricing algorithms similar to algorithms, presented in this paper, instead of auctions.

\section{References}

[AA95] Baruch Awerbuch and Yossi Azar. Blindly-competitive algorithms: Pricing and bidding a case study. Extended Abstract, April 1995.

[AD89] L.M. Ausubel and R.J. Deneckere. Reputation in bargaining and durable goods monopoly. Econometrica, 57(3):511-531, 1989.

[BDKM95] Shai Ben-David, Eyal Kushilevitz, and Yishay Mansour. Online learning versus offline learning. In P. Vitanyi, editor, Proceedings of the second EuroCOLT, pages 38-52, 1995.

[Ben89] R. Benabou. Optimal price dynamics and speculations with a storable good. Econometrica, 57:41-80, 1989.

[CBFHW93] N. Cesa-Bianchi, Y. Freund, D. P. Helmbold, and M. Warmuth. On-line prediction and conversion strategies. In John Shawe-Taylor and Martin Anthony, editors, Proceedings of EuroCOLT'93, pages 205-216, 1993.

[CLR93] T.H. Cormen, C.E. Leiserson, and R.L. Rivest. Introduction to algorithms. The MIT Press, 1993.

[EYFKT92] R. El-Yaniv, A. Fiat, R. Karp, and G. Turpin. Competitive analysis of financial games. In Proceedings of the 33rd Annual Symposium on Foundations of Computer Science, pages 327-333, 1992. 
[FNSY96] D. F. Ferguson, C. Nikolaou, J. Sairamesh, and Y. Yamini. Economic models for allocating resources in computer systems. In Scott Clearwater, editor, Marked Based Control of Distributed Systems. World Scientific Press, 1996.

[HR81] M. Harris and A. Raviv. A theory of monopoly pricing schemes with demand uncertainty. American Economic Review, 71:347-365, 1981.

[KW93] Jyrki Kivinen and Manfred K. Warmuth. Using experts for predicting continuous outcomes. In John Shawe-Taylor and Martin Anthony, editors, Proceedings of Euro COLT'93, pages 109-120, 1993.

[Mey75] R.A. Meyer. Monopoly pricing and capacity choice under uncertainty. American Economic Review, 65:326-337, 1975.

[Sob91] J. Sobel. Durable goods monopoly with with entry of new consumers. Econometrica, 59(5):1455-1485, 1991.

[Vap96] V. Vapnic. Structure of statistical learning theory. In Alex Gammerman, editor, Computational Learning and Probabilistic Reasoning, pages 3-31. John Wiley \& Sons Ltd., 1996. 


\section{A Sample Trees}

We show binary search trees that minimize the expected loss, together with the expected loss achieved, when the buyer's valuation $X$ has the uniform p.m.f. $U(a, b)$, or $X$ is the discretization (via rounding) of a continious random variable with normal p.d.f. $N(\mu, \sigma)$. From Fig. 4 , we observe that (i) The expected loss for more skewed p.d.f's is less, but the height of the corresponding tree is larger, since we can afford to have less probable $X$ at larger depths (incurring greater $\operatorname{loss}(X))$. (ii) The expected loss is an increasing function of the expected value of $X$.

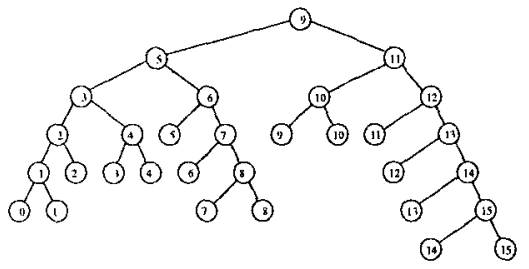

(a)
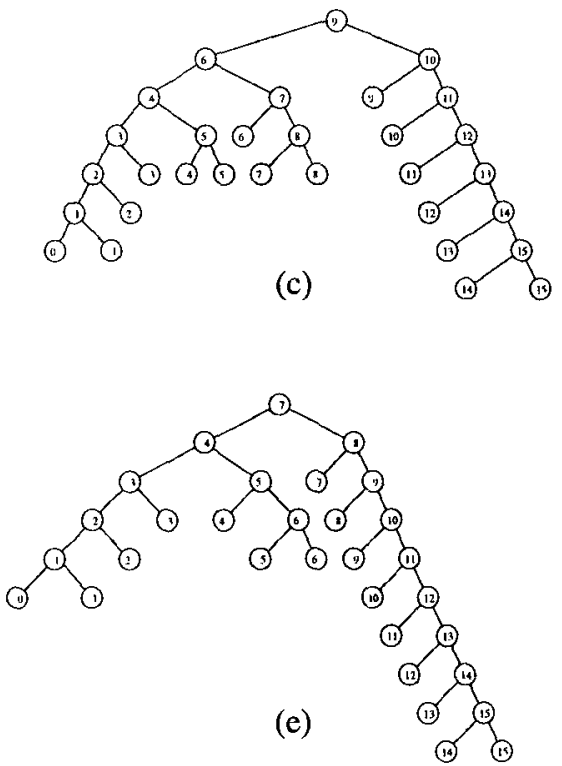
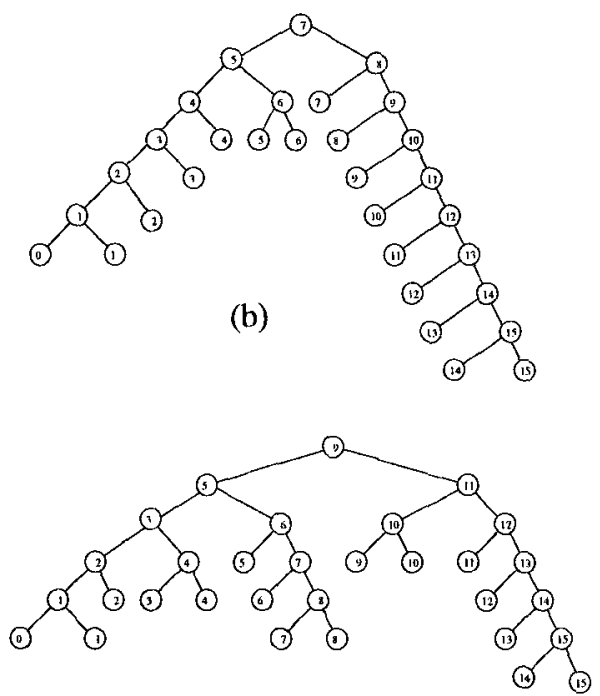

(d)

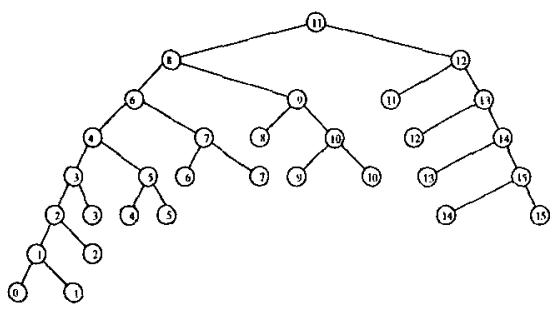

(f)

Fig. 4. (a) $X \sim U(0,15)$; exp loss $=12.5625$; (b) $X \sim N(7.5,2)$; exp_loss $=10.436841$;

(c) $X \sim N(7.5,4)$; exp_loss $=12.563369$; (d) $X \sim N(7.5,8)$; $\exp$ loss $=12.734426$;

(e) $X \sim N(4,4)$; exp_loss $=8.7110581$; (f) $X \sim N(12,4)$; exp_loss $=14.764054$. 